\title{
Site testing of the Sierra de Javalambre. First results
}

\author{
M. Moles $^{1,2}$, S.F Sánchez ${ }^{1,3,4}$, J. L. Lamadrid ${ }^{1}$, A. J. Cenarro ${ }^{1}$, D. Cristóbal-Hornillos ${ }^{1,2}$, \\ N. Maicas ${ }^{1}$, J. Aceituno ${ }^{4}$ \\ moles@cefca.es
}

Received __; accepted _

\footnotetext{
${ }^{1}$ Centro de Estudios de Física del Cosmos de Aragon (CEFCA), C/General Pizarro 1, E-41001 Teruel, Spain

${ }^{2}$ Instituto de Astrofísica de Andalucía (IAA), Consejo Superior de Investigaciones Científicas (CSIC), C/ Camino Bajo de Huétor 50, E-18008, Granada, Spain

${ }^{3}$ Fundación Agencia Aragonesa para la Investigación y el Desarrollo (ARAID)

${ }^{4}$ Centro Astronómico Hispano-Alemán, Calar Alto, (CSIC-MPG), C/Jesús Durbán Remón 2-2, E-04004 Almeria, Spain
} 


\begin{abstract}
We present the main characteristics of the Pico del Buitre, at the Sierra de Javalambre, the proposed location for the Javalambre Astrophysical Observatory. The measurements have been obtained from spectrophotometric, photometric and seeing data obtained with different monitors and instruments on the site. We have also used publicly accessible meteorological satellite data to determine the total time useful for observations.

The night-sky optical spectrum observed in a moonless night shows very little contamination by the typical pollution lines. Their contribution to the skybrightness is $\sim 0.06$ mag in $\mathrm{B}, \sim 0.09 \mathrm{mag}$ in $\mathrm{V}$ and $\sim 0.06$ mag in $\mathrm{R}$. In particular, the comparison of the strengths of the Sodium artificial and natural lines indicates that the site satisfies the IAU recommendations for a dark site. The zenithcorrected values of the moonless night-sky surface brightness are B $=22.8$ mag $\operatorname{arcsec}^{-2}, \mathrm{~V}=22.1 \mathrm{mag} \operatorname{arcsec}^{-2}, \mathrm{R}=21.5 \mathrm{mag} \operatorname{arcsec}^{-2}, \mathrm{I}=20.4 \mathrm{mag} \operatorname{arcsec}^{-2}$, which indicates that the site is very dark. The extinction has been measured for the summer period, with a typical value of $0.22 \mathrm{mag}$ in the $\mathrm{V}$-Band, with the best measured value of 0.18 mag in a totally photometric night. The median value of the seeing in the $V$-band for the last two years (2008-9) is $0.71^{\prime \prime}$, with a mode of $0.58^{\prime \prime}$. The seeing values present a seasonal pattern, being smaller in summer $\left(\sim 0.69^{\prime \prime}\right)$ than in winter time $\left(0.77^{\prime \prime}\right)$. For $68 \%$ of the analyzed nights the seeing was better than $0.8^{\prime \prime}$ during the entire night. The seeing is found to be stable for rather long periods, in particular for the nights with good seeing values. The typical scale, for nights with the seeing below $0.8^{\prime \prime}$, is about 5 hours for variations within $20 \%$ of the reference value. The fraction of totally clear nights is $\sim 53 \%$, while the fraction of nights with at least a $30 \%$ of the night clear is $\sim 74 \%$.
\end{abstract}


Subject headings: Astronomical Phenomena and Seeing 


\section{Introduction}

Any search for adequate sites for new observatories has to focus on the observing conditions such as the night sky brightness, the number of clear nights, the seeing, the transparency or the photometric stability. Looking for a potential new site in continental Spain we found in 1989 that the Sierra de Javalambre, in the province of Teruel, might be one such site. A limited number of studies and observations were accumulated at that time indicating that it was a promising site. For a variety of reasons the study had to be interrupted until recently when the idea of building a new Observatory there regained interest and new studies were funded. Consequently we have started a program to determine the main observing conditions at the proposed site for the Javalambre Astrophysical Observatory (JAO, hereafter). The selected site is on the Pico del Buitre, Sierra de Javalambre (Teruel, Spain), $1957 \mathrm{~m}$ above the sea level, at $40^{\circ} 02^{\prime} 28.67^{\prime \prime}$ North, $01^{\circ} 00^{\prime} 59.10^{\prime \prime}$ West.

The JAO will house two new telescopes to be built in the next three years. It will be run by the Centro de Estudios de Física del Cosmos de Aragón, CEFCA, recently created in Teruel. The main telescope is intended to be a large Etendue instrument for large scales surveys, with an aperture of $2.5 \mathrm{~m}$ and a FoV of 7 square degrees, with good quality over the whole field. The first Astronomical Survey is being defined to match the requirements to accurately determine the Baryonic Acoustic Oscillations along the line of sight using photometric redshifts for Luminous Red Galaxies, as proposed by the Physics of the Accelerated Universe, PAU, project. The Javalambre-PAU Astrophysical Survey will be a large scale $\left(\sim 8000\right.$ degree $\left.^{2}\right)$ photometric Survey with narrow band $(\sim 100 \AA)$ filters

covering the spectral range from $\sim 4500-8500 \AA$. The details of the project, its aims and implementation are given in (Benítez et al. 2009a).

A smaller, auxiliary telescope of $80 \mathrm{~cm}$ of aperture will be also built. The main task of 
this telescope will be to perform the required photometric calibrations for the main survey. In addition, $\sim 30 \%$ of the observing time will be accessible to the astronomical community. The telescope will have a FoV of $\sim 2$ square degrees.

In this work we study the main characteristics of the night-sky at the Pico del Buitre, identified as the best location in the Sierra de Javalambre. The study is mostly focused on the last two years (2008-09), which correspond to a period of rising solar activity from the last minimum 1 . The derived main properties have been compared with similar properties at other observatories. In Section 2 we describe the dataset collected for the current study, including a description of the data reduction process; in Section 3 we show the analysis performed on the different types of data and the results derived for each one. The main results are summarized in the Section 4.

\section{Description and Analysis of the Data}

Data from different sources were collected to characterize the different aspects of the night-sky emission at the proposed location for the JAO. These sources and the kind of data we used are described in the following subsections.

\subsection{Spectroscopic data}

A $40 \mathrm{~cm}$ aperture telescope temporarily installed at the observatory, together with a commercial DSS-7 spectrograph and a ST-8XME camera from SBIG 2 have been used to take night-sky spectra. The main characteristics of the spectrograph are listed in Table 1 .

\footnotetext{
${ }^{1}$ http://www.ngdc.noaa.gov/stp/SOLAR/ftpsolarradio.html

${ }^{2}$ http://www.sbig.com/
} 
The observations were done the night of the 23rd of August 2009, three days after the new-moon, under clear conditions. A series of exposures of 600 seconds of integration time each were taken along the night, with the telescope pointing to the Zenith and without tracking. With this setup the traces of possible astronomical sources across the slit can be easily rejected. Moreover, only the spectra without contamination by the Milky Way were considered for the analysis. The final number of spectra amounts to 14, corresponding to a total integration time of 8400 seconds.

The data were reduced using the standard procedures included in IRAF and R3D (Sánchez 2006). First, the bias was determined for each frame using the non illuminated areas of the chip and dark exposures taken at the beginning of the night with the same exposure time. The bias frame was subtracted from the corresponding science frame, which was subsequently trimmed to consider only the illuminated region. A series of dark and twilight frames were used to identify bad pixels: hot, dead and low-sensitivity ones. The values in those pixels were replaced by the median of the 5 adjacent ones along the cross-dispersion axis, in the science frames. The twilight frames were used to derive a normalization response frame, obtained dividing them by the median spectrum (i. e., the median, for each column, of the values in the corresponding rows). This frame was used to normalize the response of the detector.

For each frame the median spectrum was extracted, corresponding to a total of 40 rows. It was wavelength calibrated using the most prominent night-sky emission lines. Then the 14 frames were combined, adopting a cosmic-ray rejection scheme, to produce a single combined frame, interpolated to a common wavelength step using a simple 4-order polynomial function (eg., Sánchez 2006). The accuracy of the wavelength calibration was $\sim 1.3 \AA$, acceptable given the wavelength resolution of the spectrum for the purposes of this study. The wavelength calibrated spectrum was relative flux calibrated by adopting the 
efficiency curve published by the manufacturer. Finally, it was scaled to a typical night-sky surface brightness for an astronomical dark-site in the $V$-band.

\subsection{Sky-Brightness and Extinction data}

The night-sky surface brightness and extinction were monitored using an instrument built on purpose by J. Aceituno, the Extinction Camera and Luminance Background Register, EXCALIBUR. It is a robotic extinction monitor able to do quasi-simultaneous photometric observations in 8 bands covering the wavelength range between $3400 \AA$ to $10200 \AA$. The sky-brightness and extinction curve are derived by comparing the instrumental and catalogue photometry of pre-calibrated stars. About 16 extinction coefficients for each of the sampled bands are estimated every hour, together with up to $\sim 50$ extinction coefficients per band and per night. The same kind of instrument has been used to study the extinction curve at the Calar Alto observatory (Sánchez et al. 2007).

The instrument was operative at the mountain for a total of 13 nights, between the 23rd of July and the 5th of October 2008. For those nights, it was setup to obtain the sky-brightness at $B$ and $R$ bands, and the extinction at $\sim 500 \mathrm{~nm}$, which basically

corresponds to the $V$-band. A total of 118 estimations of the night sky surface brightness for the broad-bands, and 317 accurate estimations of the extinction for the narrow-band filters, were derived.

\section{3. $\quad$ Seeing data}

The night-sky seeing has been measured with a robotic Differential Image Motion Monitor (RoboDIMM, Aceituno 2004). This instrument measures the seeing at the wavelength corresponding to the Johnson $V$-band. It was calibrated with a similar unit 
installed at the Calar Alto observatory (Vernín \& Muñoz-Tuñoz 1995; Sánchez et al. 2007)

that was itself calibrated with a Generalized Seeing Monitor in May 2002 (Ziad et al. 2005).

The values measured by this instrument were also checked with simultaneous direct imaging obtained with the 3.5m Telescope at Calar Alto (Sánchez et al. 2008).

The instrument was first installed at the Pico del Buitre in March 2008, and it has been systematically used since then, provided that the weather conditions and the still incipient infrastructure could permit it. The data presented here include measurements up to the end of September 2009. Apart from adverse meteorological conditions, the coverage is not complete due to several kinds of accidents that affected the infrastructure.

RoboDIMM measures the transversal and horizontal components. Occasionally there is a large difference between these two values, most probably due to mechanical oscillations, that have to be eliminated from the statistics. Thanks to the inherent stability of the instrument the frequency of such departures is rather low, less than $\sim 5 \%$ in our case. The number of collected measurements amounts to 81651 in 132 nights. Despite the technical difficulties we encountered to work on the mountain, this coverage is similar to that of another well established observatories (eg., Sánchez et al. 2007).

\subsection{Satellite Information}

We used data from the satellites METEOSAT (148 images) and LANDSAT (2175 images) corresponding to the period 1983-1988 during the early site testing of the Sierra de Javalambre. They represent a faithful first approximation to the analysis of the cloud coverage in the area. Both sets of images were cross-checked and used to find the global properties of the Sierra and the differences between different peaks.

More recently, publicly available high resolution images corresponding to the period 
2005-2006 were analyzed. The data-base consist on 3,000 images from the satellite NOAA (sampling of $1.2 \mathrm{~km}$ per pixel) and 2,000 images taken with the remote sensors MODIS-TERRA and MODIS-AQUA (sampling of $250 \mathrm{~m}$ per pixel). The images correspond to different moments of the day and night, $\mathrm{UT}=0 \mathrm{~h}, 06 \mathrm{~h}, 12 \mathrm{~h}$ and $18 \mathrm{~h}$. Terra MODIS and Aqua MODIS are viewing the entire Earth's surface every 1 to 2 days, acquiring data in 36 spectral bands covering the wavelength range between $0.4 \mu \mathrm{m}$ to $14.4 \mu \mathrm{m}$. These images can be used to determine the presence of clouds at a given location, on the basis of the difference of temperature between the surface and the clouds, provided by the infrared measurements. The analysis was done on images of $20 \times 20 \mathrm{~km}$ centered on the Pico del Buitre.

\section{Results and Discussions}

We describe here the analysis performed over each of the different sets of data.

\subsection{Night Sky Spectrum}

Figure 1 shows the night-sky spectrum obtained as explained before. This is the first time that a spectrum of the night-sky over Javalambre is published. The night-sky emission lines have been labeled with its name and wavelength. For comparison purposes, published night-sky spectra corresponding to the Calar Alto and the Kitt Peak observatories have been included in the figure.

The main natural contribution to the night-sky light is airglow. It produces prominent lines like OI $\lambda \lambda 5577,6300 \AA$, the $\mathrm{OH}$ bands in the red and NIR and a pseudo-continuum in the blue (due to overlapping $\mathrm{O}_{2}$ bands, 2600-3800 $\AA$ ), and in the green $\left(\mathrm{NO}_{2}\right.$ bands, 5000-6000 $\AA$ ). It also contributes to the ubiquitous NaD $\lambda 5890 / 6 \AA$ doublet, a line that can 
be contaminated by light-pollution from low and high pressure street-lamps (see Figure 1). A more detailed description of the effects of the airglow on the night-sky emission can be found in Benn \& Ellison (1998a). An atlas of the airglow from $3100 \AA$ to $10000 \AA$ was presented by Ingham (1962) and Broadfoot \& Kendell (1968). Other contributions to the night-sky spectrum are the zodiacal light, the starlight, and the extragalactic light, which increase the background continuum emission (see Benn \& Ellison 1998a and references therein).

Apart form the natural contributions, the night sky spectrum can be affected by light-pollution, mostly from street-lights in populated areas near to observatories. Light-pollution arises principally from tropospheric scattering of light emitted by Sodium (high and low pressure), Mercury-vapor and incandescent street lamps (McNally 1994; Osterbrock et al., 1976; Holmes 1997). The Na low pressure lamps are those with less impact on astronomical observations, since it produces most of its light concentrated in the $5890 / 6 \AA$ and $8183 / 95 \AA$ lines. High-pressure sodium lamps emit most of its light in a broad, FWHM $\sim 400 \AA$ Na line centered at $\sim 5890 \AA$ that shows a central reversal. They also show strong emission at $8183 / 95 \AA$ and fainter emission lines. The Mercury lamps produce narrow lines at 3651/63 $, 4047 \AA, 4358 \AA, 5461 \AA, 5770 \AA$ and $5791 \AA$, together with broad features at $6200 \AA$ and $7200 \AA$, FWHM 100 $\AA$, from the phosphor used to convert UV to visible light. They also produce a weak continuum emission over the whole visible range.

The spectrum of the incandescent lamps consists of continuum emission only, and it is difficult to identify in a night-spectrum. Finally, the high-pressure metal halide lamps, nowadays frequently used in the illumination of sport stadiums and monuments, exhibit some Scandium, Titanium and Lithium emission lines, that are characterized by a blue edge due to molecular bands (General Electric 1975; Lane \& Garrison 1978; Osterbrock et 
al. 1976).

The night-sky spectrum of the Pico del Buitre illustrated in Figure 1 shows little evidence of significant light pollution from street-lamps. The Mercury lines, that are present in the night-sky spectrum of the Calar Alto and Kitt-Peak observatories, are not detected. Moreover, the broad emission at $\sim 5900 \AA$ from high-pressure sodium lamps is significantly weaker than in these two observatories, whereas the potentially strong emission line at $5893 \AA$ from low-pressure sodium lamps is barely detected at most. The detected contamination, that includes the Sc line at $5351.1 \AA$, is dominated by the illumination of the city and metropolitan area of Valencia ( $\sim 810,000$ habitants), at $\sim 100 \mathrm{~km}$ towards the South-East. In this regards the night-sky spectrum is more similar to that of the Observatory of the Roque de los Muchachos, at La Palma, published by Pedani (2005).

To quantify the contribution of the light pollution to the night-sky spectrum of the Pico del Buitre we have determined the flux intensity corresponding to each detected emission feature using the procedure described in Sánchez et al. (2007). The values we have found are listed in Table 2, We also give in the Table the flux from the Sodium broad-band emission, estimated by fitting the observed feature with a single broad gaussian function. All the fluxes were converted to Rayleigh following the conversion formulae by Benn \& Ellison (1998a).

Quantifying the artificial contribution to the Sodium lines is difficult because of the natural contribution to them. From the analysis by Benn \& Ellison (1998a) this natural contribution to the broad Sodium emission band can be estimated to be $\sim 0.04$ mag in the $\mathrm{V}$ and $\mathrm{R}$ bands. Adopting this value, the contribution from the artificial component can be easily estimated. The results are listed in Table 3 for the $B, V$ and $R$ bands, and for a set of medium band filters used by the ALHAMBRA survey (Moles et al., 2008; Benítez et al., 2009b). They are included to illustrate the effects of the pollution lines 
when median/narrow-band filters, as proposed for large-scale surveys (see Benítez et al., 2009b), are used. The results show that the contribution to the natural component at the Javalambre site in all bands is small. In fact, the site of Pico del Buitre satisfies the IAU criterium for a dark site since the artificial contribution to the Sodium emission, estimated to be $\leq 0.06$ mag, doesn't exceed that of the natural airglow (Smith 1979).

A long term monitoring of the night-sky spectrum at the observatory will be required to analyze the evolution of the light pollution along the time. Most major observatories nearby heavily populated areas have some kind of night-sky protection laws. Although the Sierra de Javalambre site does not benefit yet from any local sky-protection law to regulate the street illumination, it appears that its effect is low in the night-sky spectrum. In any case a proper protection law would be most convenient to preserve the quality of the site.

\subsection{Night Sky Brightness and Atmospheric Extinction}

The data from EXCALIBUR were used to determine the night sky brightness in the $B$ and $R$ bands and the extinction in the $V$ band. The sky brightness measurements were not corrected for extinction, following the convention adopted in most of the recent studies of sky brightness (e.g., Walker 1988b; Krisciunas 1990; Lockwood et al 1990; Leinert et al. 1995; Mattila et al. 1996; Benn \& Ellison 1998a,b, Sánchez et al. 2007). They are only corrected to the zenith using the expression by Patat (2003),

$$
\Delta m=-2.5 \log _{10}[(1-f)+f X]+\kappa(X-1)
$$

where $\Delta m$ is the increase in sky brightness at a given band for an airmass $X, f$ is the fraction of the total sky brightness generated by airglow, being (1- $f$ ) the fraction produced outside the atmosphere (hence including zodiacal light, faint stars and galaxies) and $\kappa$ is 
the extinction coefficient at the corresponding wavelength. To apply that formula we have used the average extinction along each night and a typical value of $f=0.6$ (Patat 2003; Sánchez et al. 2007). The collected data spans two complete moon phases, having a good coverage of the different phases/illumination of the moon.

Table 4 lists the mean values of the sky brightness for dark nights, i. e., with Moon illumination less than $5 \%$. It contains the measured $B$ and $R$ values and the estimated values for the $V$ and $I$ bands. The later were obtained from the night-sky spectrum scaled to the $B$ and $R$ values measured for dark nights. In the same table values for other observatories are given. It appears that Javalambre is one of the darkest observatories even if it has to be taken into account that some of the data in the Table (for example for Paranal) were obtained during the maximum of solar activity.

The measurements of the extinction obtained for Javalambre correspond to the summer season when higher than year average extinction values are expected. This is due to the presence of aerosols and, very occasionally at the Javalambre latitude, to dust from the Sahara desert. The overall effect is clearly seen in the seasonal evolution of the dust extinction at well established observatories in Spain, like La Palma (Benn \& Ellison 1998a) or Calar Alto (Sánchez et al. 2007).

Considering all the 317 individual measurements of the extinction coefficient provided by EXCALIBUR the median value amounts to 0.27 mag, with a high dispersion of 0.14 mag. Inspecting the data set we verify that 4 nights have very high extinction coefficients, over 0.4 mag. These were highly non-photometric nights with a very unusual dust content in the atmosphere. Excluding them, the median value for $\mathrm{k}_{V}$ is now 0.22 , much closer to the expected values and very similar to that of the summer period in Calar Alto. Indeed, truly photometric nights are characterized by rather small extinction variations along the night. For the best night in our sampling, October 5, 2008, the variations of $\mathrm{k}_{V}$ are within $15 \%$ and 
the night extinction coefficient is $\sim 0.17 \pm 0.03 \mathrm{mag}$, a value consistent with that reported for the winter season in other major observatories, when the aerosol contribution is lower (Benn \& Ellison 1998b; Sánchez et al. 2007). Despite of that, the average extinction values reported so far seem to be rather high compared with the statistical values reported in other observatories. A visual inspection of the extinction curves derived by EXCALIBUR for different wavelengths for the different nights (eg., Sánchez et al. 2007), points to an excess of Ozone absorption, rather than a high aerosol content in the vicinity of the observatory. The limited amount of data collected so far does not allow us to perform a more detail analysis, that will require a larger dataset. For similar reasons, no seasonal pattern in the extinction was analyzed due to the short time coverage of the collected data.

\subsection{Atmospheric Seeing}

The seeing data described in section 2.3 were used to determine an average seeing for each monitored night along the $\sim 1.5$ years covered by the data discussed here. In Figure 2 the nightly measured data points are shown. The obvious lack of data for some periods is due to technical problems related to the rather precarious situation we still have at the mountain to get the monitors working and safe from accidents. In fact the two longer periods without data do correspond to two major events: damage by a strong wind and a robbery. Looking at the nightly average values (Figure 21), we see that $\sim 68 \%$ of the nights have median seeing below $0.8^{\prime \prime}$. Considering the whole data set of all the validated measurements, amounting to $\mathrm{N}=81651$ data points, the median value we find is $0.71^{\prime \prime}$. The distribution is shown in Figure 3. It can be seen that the mode is $0.58^{\prime \prime}$ and more than $85 \%$ of the values are below $1^{\prime \prime}$.

The trend of the average nightly values that can be appreciated in Figure 2 is suggestive of a seasonal pattern, as it is found in other places (Sánchez et al. 2007). To further 
investigate this possibility we have built two subsets of data, one for May-September (Summer period) and the other for October-April (Winter season). With some caution due to the lack of the data for January and February, its is clear from Figure 3 the seeing for the Summer period (median value of $0.69^{\prime \prime}$ ) is better than for the Winter period (median $\left.0.77^{\prime \prime}\right)$. Not only the median seeing is better in the Summer season, but the chances of having better seeing in a Summer night are much higher.

In Table 5 we have collected the median seeing determined for Javalambre and published data for other observatories. It appears that the median seeing at Javalambre is comparable to that of the most highly reputed observatories in the world.

We have looked for the possible dependence of the seeing value with some meteorological parameters. We have found strong indication of correlation only with the wind speed and direction. We have selected all the seeing measurements that are coincident within 5 minutes with measurements of the wind direction, a total of 16542 data points. We find that the seeing worsens for wind speeds over $18 \mathrm{~m} / \mathrm{s}$, a completely expected result. More interesting is the relation between seeing quality and wind direction. Figure 4 shows the median seeing for different wind directions. It is clear that the dominant winds are from western directions and the best seeing values are found under these wind conditions $\left(\right.$ median $\left.=0.60^{\prime \prime}\right)$. The worst seeing values are found when the wind comes from the North or North-East directions $\left(\sim 1^{\prime \prime}\right)$. Fortunately these are the less frequent wind directions.

We have also considered the stability of the seeing. Inspecting the data we find that the seeing can be very stable. Indeed, it is frequent to find nights, particularly under good seeing conditions $\left(<0.8^{\prime \prime}\right)$ presenting seeing variations of just a few per-cent. To characterize the stability of the seeing on an objective basis we have worked out the typical time scales to have the same seeing within some percentage of the reference value. For that we have analyzed the whole data set computing the point-to-point variations within 
a given percentage. Then the distribution of the resulting time scales for every percentage were computed. The result is plotted in Figure 5. We find that the time scale for the seeing stability is longer for better seeing values. It can be as long as 5 or more hours for variations up to $20 \%$ for reference seeing values below $0.8^{\prime \prime}$.

\subsection{Fraction of Useful Time}

The analysis of the low resolution images from METEOSAT and LANDSAT for the period 1983-1988 showed that the fraction of completely clear days was about 53\%. They also showed that the SE side of the Sierra de Javalambre has the best statistics so all the efforts were subsequently concentrated on the Pico del Buitre, the highest peak in that specific area.

The analysis of the high resolution images corresponding to the period 2005-2006 confirms the earlier values, indicating that no long-term evolution in the overall conditions has occurred. It is confirmed that the number of totally clear nights per year amounts to 193 or $\sim 53 \%$. Another important result is that the number of nights with less than $50 \%$ cloud cover amounts to $62.2 \%$ (227 nights per year). We notice that these figures compare very well with highly reputed Observatories around the world (see for example, Webster 2004; Sánchez et al. 2007).

The fraction of useful time has a clear seasonal pattern, being higher in Summer than in Winter. We show in Figure 6 the fraction of time with cloud coverage lower than a certain limit $(10 \%, 50 \%$ and $70 \%$ of the time, for each night), for the different months along a year. The time period between June and August has the better weather statistics, with $\sim 60 \%$ of totally clear nights. On the other hand, the worst months are March, May and November, with $\sim 35 \%$ of clear nights. These months are traditionally the periods with the 
largest precipitation values in continental Spain.

\section{Conclusions}

We have characterized the main properties of the night-sky at the Pico del Buitre, Sierra de Javalambre (Teruel, Spain), the site proposed for the Javalambre Astrophysical Observatory. Data taken with seeing, extinction and night-sky brightness in situ monitors along a period of 1.5 years, together with satellite high spatial resolution meteorological data for the years 2005 and 2006 has been used to determined the relevant parameters for optical observations. The main conclusions that have been reached are:

- A night-sky spectrum, covering the wavelength range form $3950 \AA$ to $8150 \AA$, for the moonless dark-time at the observatory has been presented for the first time. Airglow and light-pollution emission lines are detected in this spectrum. The strength of the light-pollution lines has been measured, estimating their contribution to the emission in different bands. The light pollution is weak or absent (for the Mercury lines, for example). The Pico del Buitre fulfills the IAU recommendations for a dark astronomical site (Smith 1979).

- The moonless night-sky brightness at the zenith has been measured in the $B$ and $R$ bands, and deduced for the $V$ and $I$-bands. It is found that the site is particularly dark with marginal contribution at most by artificial pollution.

- The extinction, estimated for the Summer season, shows a typical value of $k_{V}=0.22$ mag, similar to that of other observatories for the same season. The extinction found for a photometric night was $k_{V} \sim 0.17 \mathrm{mag}$, consistent with the expected value for a night without aerosols. 
- The median seeing along the last 1.5 years was $0.71^{\prime \prime}$, with $68 \%$ of the nights with $<0.8^{\prime \prime}$ median value. The seeing shows a seasonal dependence being better in the Summer period. These values put the Pico del Buitre among the first rank known observatories.

The seeing shows a clear dependency with the wind direction. Only for $\mathrm{N}$ and NE directions the seeing increases to near $1^{\prime \prime}$. For all the other directions of the wind rose the typical value is $\sim 0.60^{\prime \prime}$. The frequency of the N-NE wind direction is less than $10 \%$.

The seeing at the observatory is remarkably stable. Our measurements indicate that in good seeing conditions $\left(<0.8^{\prime \prime}\right)$, the seeing can be stable for more than 5 hours within $20 \%$ of the reference value.

- The fraction of completely clear (less than $10 \%$ cloud coverage) nights amounts to $53 \%$. The fraction of useful nights (at least a $30 \%$ of the night clear), amounts to 74\%. So far, the fraction of photometric nights is still not known for the observatory, with the current data.

We conclude that the Pico del Buitre, the proposed site to build the Javalambre Astrophysical Observatory is a particularly good astronomical site. The fact that Javalambre doesn't present special accessing difficulties and that it is placed in continental Europe represents an added advantage from the point of view of building and operation.

\section{Acknowledgments}

The Ministerio de Ciencia e Innovación, the Consejo Superior de Investigaciones Científicas and the Departamento de Ciencia, Innovación y Universidad of the Gobierno de Aragón are acknowledged for support to the site testing work and the JAO project. 
SFS thanks the Spanish Plan Nacional de Astronomía program AYA2005-09413-C02-02, of the Spanish Ministery of Education and Science and the Plan Andaluz de Investigación of Junta de Andalucía as research group FQM322.

We thanks the Calar Alto Observatory, and in particular his director, Dr. J. Alves, to allow us to use their spare DIMM for the first 6 months of 2008 to start this study. We also thanks the Instituto Tecnológico de Aragón for help with the management of the site testing and technical advice.

We thank Dr. J. Muñoz, Dr. M. A. Torrejón, J. A. Quesada and S. Abad for their help during the early studies at Pico del Buitre. We also thank E. Esco for his support and involvement at the beginning of the new period of measurements. We also thank the members of the Amateur Astronomer Society in Teruel (ACTUEL), F. García, M. Martín, J.A. Ortíz and J. Bullón, for the help with some of the observations.

We also thanks for Dr. J. Melnick anf Dr. M. Sarazon for their advice in the preparation of this study. 


\section{REFERENCES}

Aceituno, J., 2004, Calar Alto Newsletter n.8, http://www.caha.es/newsletter/news04b/Aceituno/Newsletter.html

Benítez, N., et al., 2009, ApJ, 691, 241

Benítez, N., et al. 2009, ApJ, 692, L5

Benn, C.R., Ellison, S.L., 1998, La Palma Techincal Note, 115.

Benn, C. R., \& Ellison, S. L. 1998, New Astronomy Review, 42, 503

Broadfoot, A.L., \& Kendall, K.R., 1968, J. Geophys. Res., 73, 426

General Electric Co. 1975, High Intensity Discharge Lamps, Booklet TP-109R

Holmes, R.W., 1997, Observatory, 117, 25

Ingham M.F, 1962, MNRAS, 124, 505

Krisciunas, K., 1990, PASP, 102, 1052

Lane, M. C., \& Garrison, R. F. 1978, JRASC, 72, 198

Leinert, C., Vaisanen, P., Mattila, K., \& Lehtinen, K. 1995, A\&AS, 112, 99

Lockwood, G.W., Floyd, R.D., Thompson, D.T., 1990, PASP, 102, 481

Massey, P., Abraham, T., Bohannan, B., Claver, C., Green, R., Jacoby, G., Wolff, R., 2000, NOAO Newsletter, KPNO Operations, March 2000, Number 61

Mattila K., Väisänen P., v. Appen-Schuur G.F.O., 1996, AAS, 119, 153

McNally, D., 1994, ed. 'The Vanishing Universe - Adverse Entironmental Impacts on Astronomy' (Cambridge University Press 1994). 
Moles, M., et al. 2008, AJ, 136, 1325

Murtagh, F., \& Sarazin, M. 1993, PASP, 105, 932

Muñoz-Tuñon, C., Vernin, J., \& Varela, A. M. 1997, A\&AS, 125, 183

Osterbrock, D. E., Walker, M. F., \& Koski, A. T. 1976, PASP, 88, 349

Patat, F. 2003, A\&A, 400, 1183

Pedani, M. 2009, PASP, 121, 778

Pilachowski, C. A., Africano, J. L., Goodrich, B. D., \& Binkert, W. S. 1989, PASP, 101, 707

Racine, R. 1989, PASP, 101, 436

Sánchez, S. F., 2006, AN, 327, 850

Sánchez, S. F., Aceituno, J., Thiele, U., Pérez-Ramírez, D., \& Alves, J. 2007, PASP, 119, 1186

Sánchez, S. F., Thiele, U., Aceituno, J., Cristobal, D., Perea, J., \& Alves, J. 2008, PASP, 120,1244

Sarazin, M., Melnick, J., Navarrete, J., \& Lombardi, G. 2008, The Messenger, 132, 11

Smith, F.G., 1979, Trans IAU, 17A, 220

Taylor, V. A., Jansen, R. A., \& Windhorst, R. A. 2004, PASP, 116, 762

Vernin, J., \& Muñoz-Tuñon, C. 1995, PASP, 107, 265

Walker, A., 1987a, NOAO Newsletter, No. 10, 16

Walker, A., 1988a, NOAO Newsletter, No. 13, 22 
Walker G., 1987b, Astronomical Observations, Cambridge Uni. Press, p. $47 f f$

Walker M.F., 1988b, PASP, 100, 496

Walker, A., Schwarz, H.E., 2007, http://www.ctio.noao.edu/site/pachon_sky/

Webster, 2004, DECam Note, Version 2.0, Feb. 20, 2004.

Ziad, A., et al. 2005, MNRAS, 362, 455 
Table 1: Main properties of the DDS-7 Spectrograph

\begin{tabular}{ll}
\hline \hline Parameter & Value \\
\hline Slit-Width & $\sim 2.7^{\prime \prime}$ \\
Slit-Length & $\sim 19^{\prime \prime}$ \\
Spectral Resolution (FWHM) & $15 \AA$ \\
Spectral Sampling & $5.4 \AA /$ pixel \\
Spectral Range & $4000-8100 \AA$ \\
Peak Efficiency & 0.4 \\
Wavelength of the Peak Efficiency & $6000 \AA$ \\
\hline
\end{tabular}


Table 2: Properties of the detected emission lines

\begin{tabular}{|c|c|c|c|}
\hline Line Id & $\begin{array}{l}\text { Wavelength } \\
(\AA)\end{array}$ & Flux* & $\begin{array}{r}\text { Flux }^{* *} \\
\mathrm{R}\end{array}$ \\
\hline $\mathrm{HgI}$ & 4827,32 & $0.4 \pm 0.1$ & 2.9 \\
\hline $\mathrm{NaI}$ & 4978,83 & $1.2 \pm 0.1$ & 8.6 \\
\hline $\mathrm{NaI}$ & 5149,53 & $0.4 \pm 0.1$ & 2.9 \\
\hline $\mathrm{ScI}$ & 5351.1 & $0.7 \pm 0.1$ & 5.0 \\
\hline $\mathrm{HgI}$ & 5461 & $0.9 \pm 0.1$ & 6.5 \\
\hline $\mathrm{OI}$ & 5577 & $6.8 \pm 0.1$ & 48.8 \\
\hline $\mathrm{NaI}$ & 5683,88 & $1.2 \pm 0.1$ & 8.6 \\
\hline $\mathrm{HgI}$ & 5770,91 & $0.5 \pm 0.1$ & 3.6 \\
\hline Broad NaD & 5893 & $6.3 \pm 0.2$ & 45.2 \\
\hline $\mathrm{NaI}$ & 6154,61 & $0.8 \pm 0.1$ & 5.7 \\
\hline $\mathrm{OI}$ & 6300 & $2.1 \pm 0.1$ & 15.1 \\
\hline OI & 6364 & $0.9 \pm 0.1$ & 6.5 \\
\hline
\end{tabular}


Table 3: Contribution of the light pollution lines to the sky-brightness

\begin{tabular}{ll}
\hline \hline Band & $\Delta \mathrm{mag}$ \\
\hline$B$ & 0.06 \\
$V$ & 0.09 \\
$R$ & 0.06 \\
$4280 / 331$ & 0.04 \\
$5510 / 331$ & 0.05 \\
$5820 / 331$ & 0.22 \\
\hline \hline
\end{tabular}


Table 4: Summary of the night-sky surface brightness

\begin{tabular}{llllll}
\hline \hline Site \& Date & $\mathrm{B}$ & $\mathrm{V}$ & $\mathrm{R}$ & $\mathrm{I}$ & Reference \\
\hline Javalambre & $22.8 \pm 0.6$ & $22.1 \pm 0.5$ & $21.5 \pm 0.3$ & $20.4 \pm 0.5$ & This work \\
\hline \hline La Silla 1978 & 22.8 & 21.7 & 20.8 & 19.5 & Mattila et al. (1996) \\
Kitt Peak 1987 & 22.9 & 21.9 & & & Pilachowski et al. (1989) \\
Cerro Tololo 1987 & 22.7 & 21.8 & 20.9 & 19.9 & Walker (1987a,88a) \\
Calar Alto 1990 & 22.6 & 21.5 & 20.6 & 18.7 & Leinert et al. (1995) \\
La Palma 1990-92 & 22.5 & 21.5 & & & Benn \& Ellison (1998a,b) \\
La Palma 1994-96 & 22.7 & 21.9 & 21.0 & 20.0 & Benn \& Ellison (1998a,b) \\
Mauna Kea 1995-06 & 22.8 & 21.9 & & & Krisciunas (1997) \\
Paranal 2000-01 & 22.6 & 21.6 & 20.9 & 19.7 & Patat et al. (2003) \\
MtGraham 2000-01 & 22.86 & 21.72 & 21.19 & & Taylor et al. (2004) \\
Cerro Pachón 2005 & 22.43 & 21.63 & & 20.3 & Walker \& Schwarz (2007) \\
Calar Alto 2007 & 22.86 & 22.01 & 21.36 & 19.25 & Sánchez et al. (2007) \\
MtGraham 2008 & 22.81 & 21.81 & 20.82 & 19.78 & Pedani (2009) \\
\hline
\end{tabular}


Table 5: Median seeing in the $V$-band compared with other astronomical sites

\begin{tabular}{lcl}
\hline \hline Site & Median seeing & Reference \\
\hline Javalambre & $0.71^{\prime \prime}$ & This work \\
Javalambre (Winter) & $0.77^{\prime \prime}$ & “ “ \\
Javalambre (Summer) & $0.69^{\prime \prime}$ & “ “ \\
\hline Mauna Kea (1987) & $0.50^{\prime \prime}$ & Racine (1989) \\
Paranal (1993) & $0.64^{\prime \prime}$ & Murtagh \& Sarazin (1993) \\
Paranal (2002-2007) & $0.65^{\prime \prime}$ & Sarazin et al. (2008) \\
La Palma (1997) & $0.76^{\prime \prime}$ & Muñoz-Tuñon et al. (1997) \\
La Silla (1999) & $0.79^{\prime \prime}$ & ESO webpage* \\
Paranal (2005) & $0.80^{\prime \prime}$ & ESO webpage* \\
La Silla (1993) & $0.87^{\prime \prime}$ & Murtagh \& Sarazin (1993) \\
Calar Alto (2006-2007) & $0.90^{\prime \prime}$ & Sánchez et al. (2007) \\
MtGraham (1999-2002) & $\sim 0.97^{\prime \prime}$ & Taylor et al. (2004) \\
Paranal (2006) & $\sim 1.00^{\prime \prime}$ & ESO webpage** \\
KPNO (1999) & $\sim 1.00^{\prime \prime}$ & Massey et al. (2000) \\
Lick (1990-1998) & $\sim 1.90^{\prime \prime}$ & MtHamilton webpage \\
\hline \hline
\end{tabular}

(*) http://www.ls.eso.org/lasilla/seeing/

(**) http://www.eso.org/gen-fac/pubs/astclim/paranal/seeing/adaptive-optics/statfwhm.html

$\left.{ }^{* * *}\right)$ http://www.eso.org/gen-fac/pubs/astclim/paranal/seeing/singstory.html

$(* * * *)$ https://mthamilton.ucolick.org/techdocs/MH_weather/obstats/seeing.html 


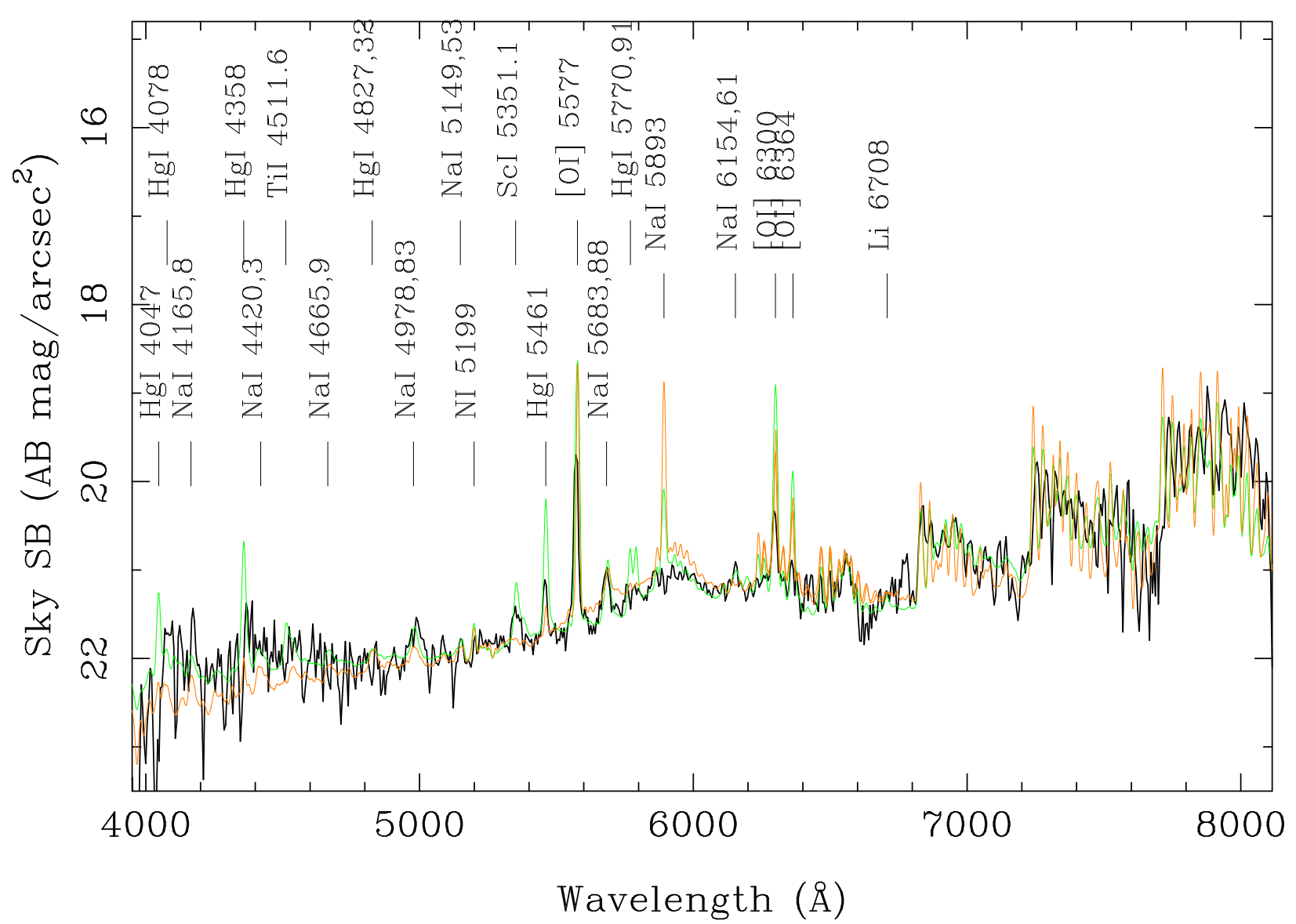

Fig. 1.- Night-sky spectrum at Pico del Buitre, Sierra de Javalambre, in the optical wavelength range (3700-7950 AA), obtained the night of the 23rd of August 2009, with dark-time and photometric conditions (black-line). The intensity has been scaled to that of the typical moonless night sky-brightness in the $V$-band. The most important night-sky emission lines have been labelled even if they are not present in the spectrum. The NaI broad emission centered at $\sim 5900 \AA$, and the water vapor Meinel bands are clearly identified in the spectrum. For comparison purposes we have included the night sky spectrum at the Calar Alto Observatory (green line, Sánchez et al. 2007) and that of the Kitt Peak observatory (orange line, Massey \& Foltz 2000). The spectral resolution and the sky-brightness at $5000 \AA$, have been scaled to match our data. The low intensity of the pollution lines at Pico del Buitre is clearly appreciated in this Figure (eg., the NaI 5893 Åand Hg 5770,91 Ålines). 


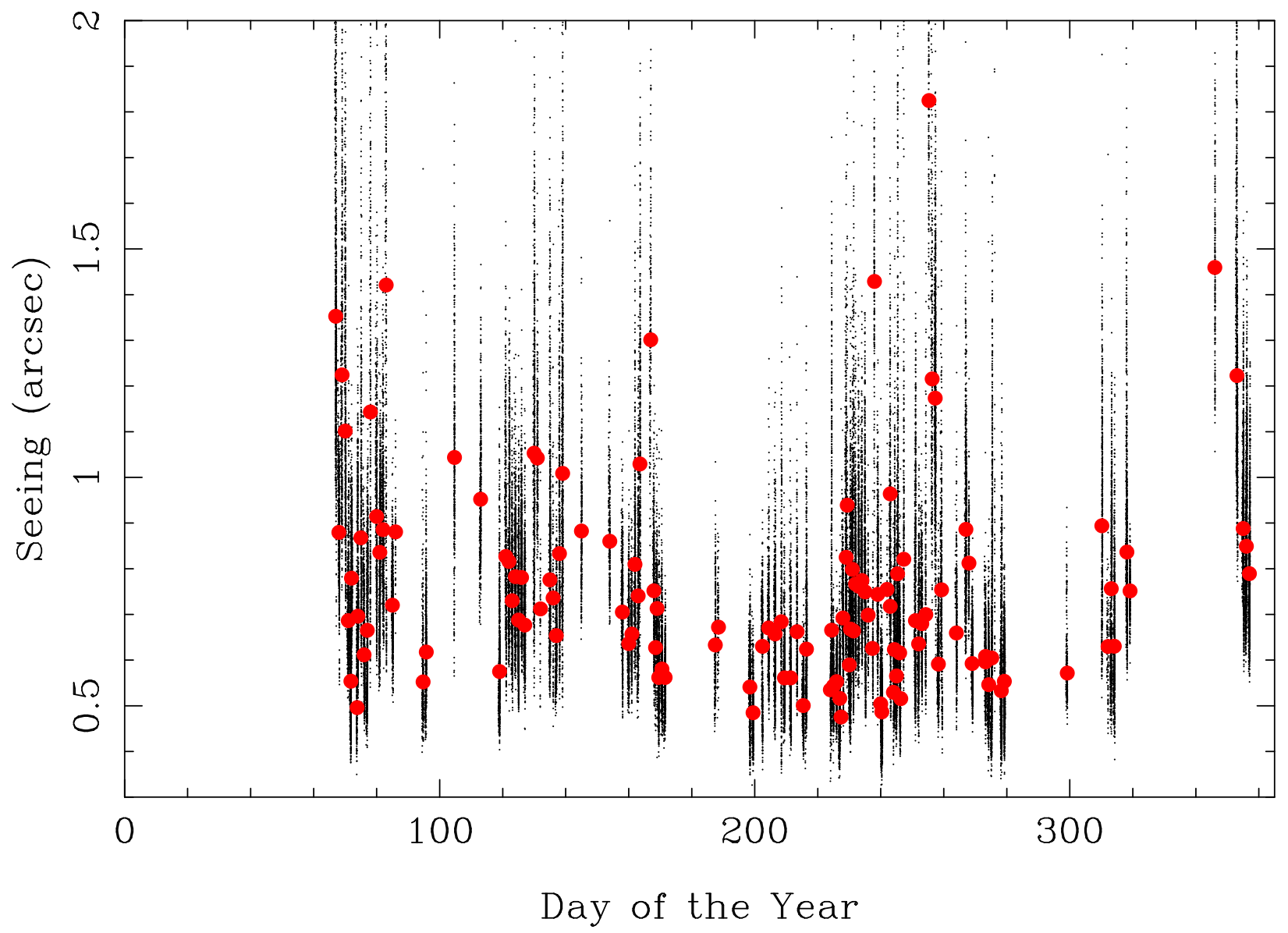

Fig. 2.- Distribution of the seeing collected for the 132 nights from March 2008 to September 2009 with RoboDIMM data, along the day of the year. The black-dots shows the 81651 individual data points, and the red-solid circles the median value for each night. It is appreciated a considerable coverage of the year, appart from January and February. 


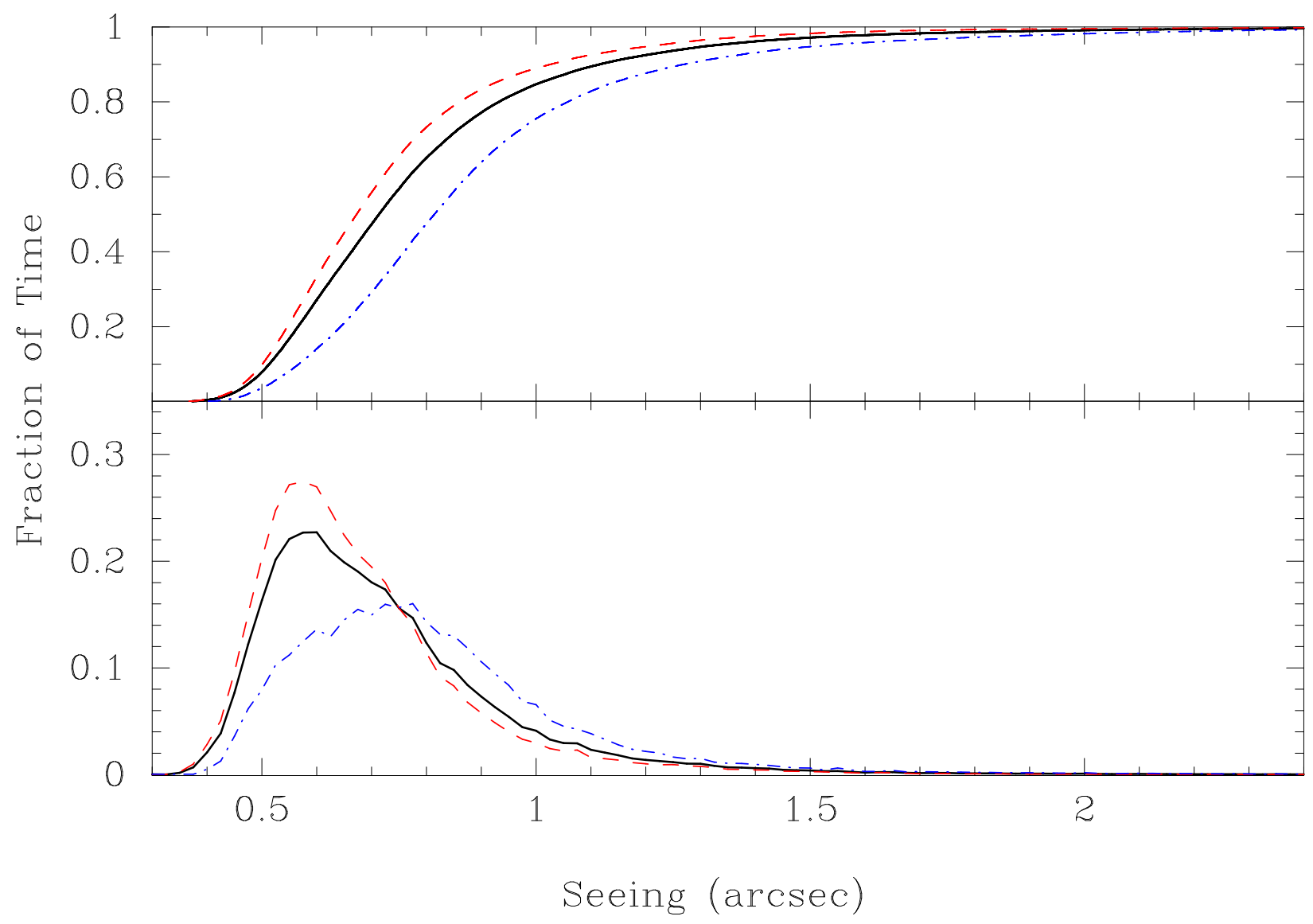

Fig. 3.- Top panel: Cumulative distribution of the seeing values shown in Fig. 2, normalized to one (solid line), together with similar distributions for the seeing data corresponding to the Summer (red dashed-line), and Winter (blue dashed-dotted line) seasons. Bottom panel: Normalized histogram of the cumulative distributions shown in the top panel (same symbols). The median value of the seeing is $\sim 0.71^{\prime \prime}$, with $\sim 68 \%$ of the nights with the median seeing below $0.8^{\prime \prime}$. The distributions show that the seeing tends to be better in Summer than in Winter. 


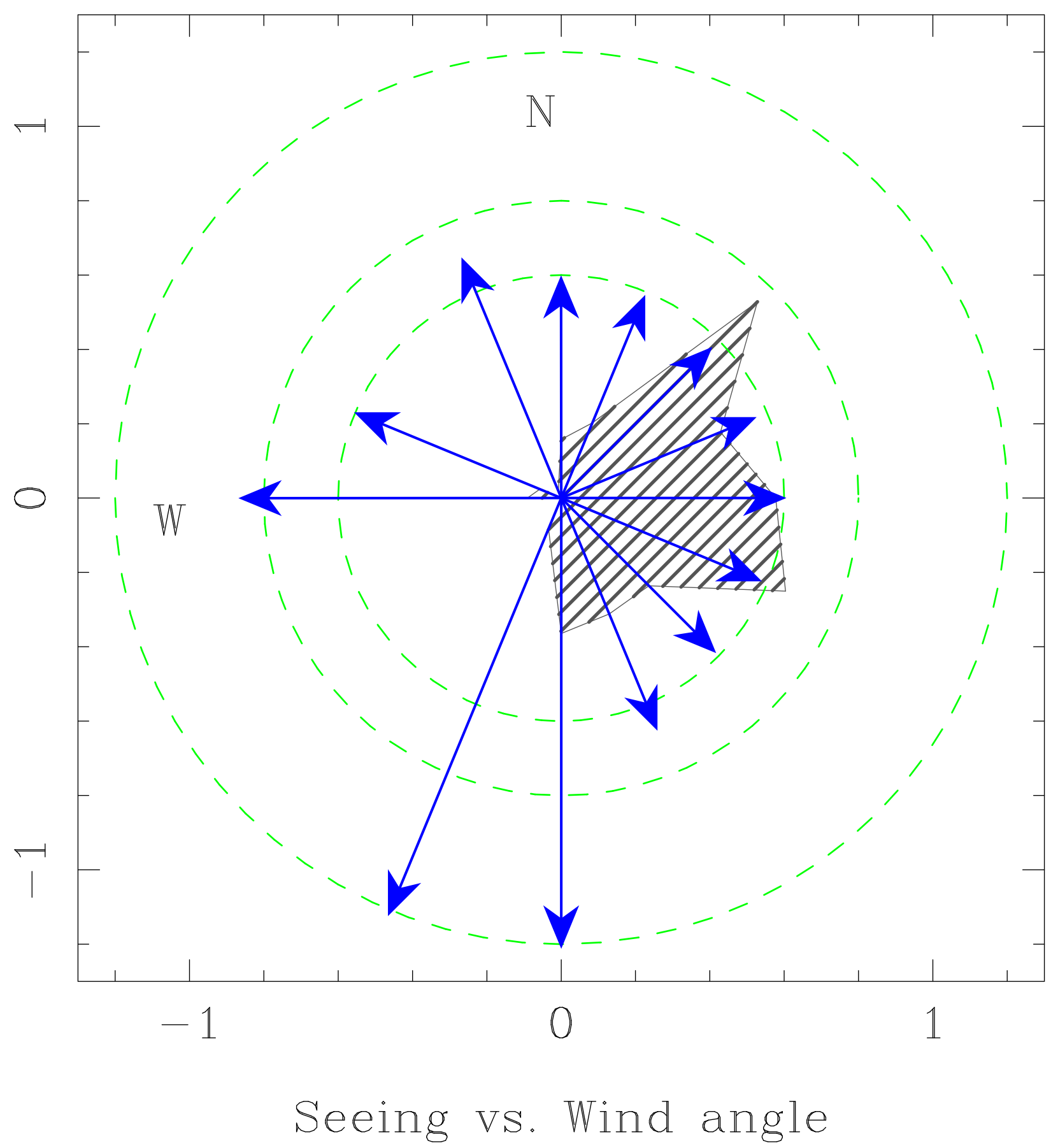

Fig. 4.- Seeing distribution for different wind directions. Each arrow indicates the wind direction and its length gives the corresponding typical seeing value. The dashed-line circles indicates three different seeing values, $0.6^{\prime \prime}, 0.8^{\prime \prime}$ and $1.2^{\prime \prime}$. The polygonal line indicates the relative frequency of each wind direction, normalized to one and scaled by a factor 5 to make it more clearly visible. 


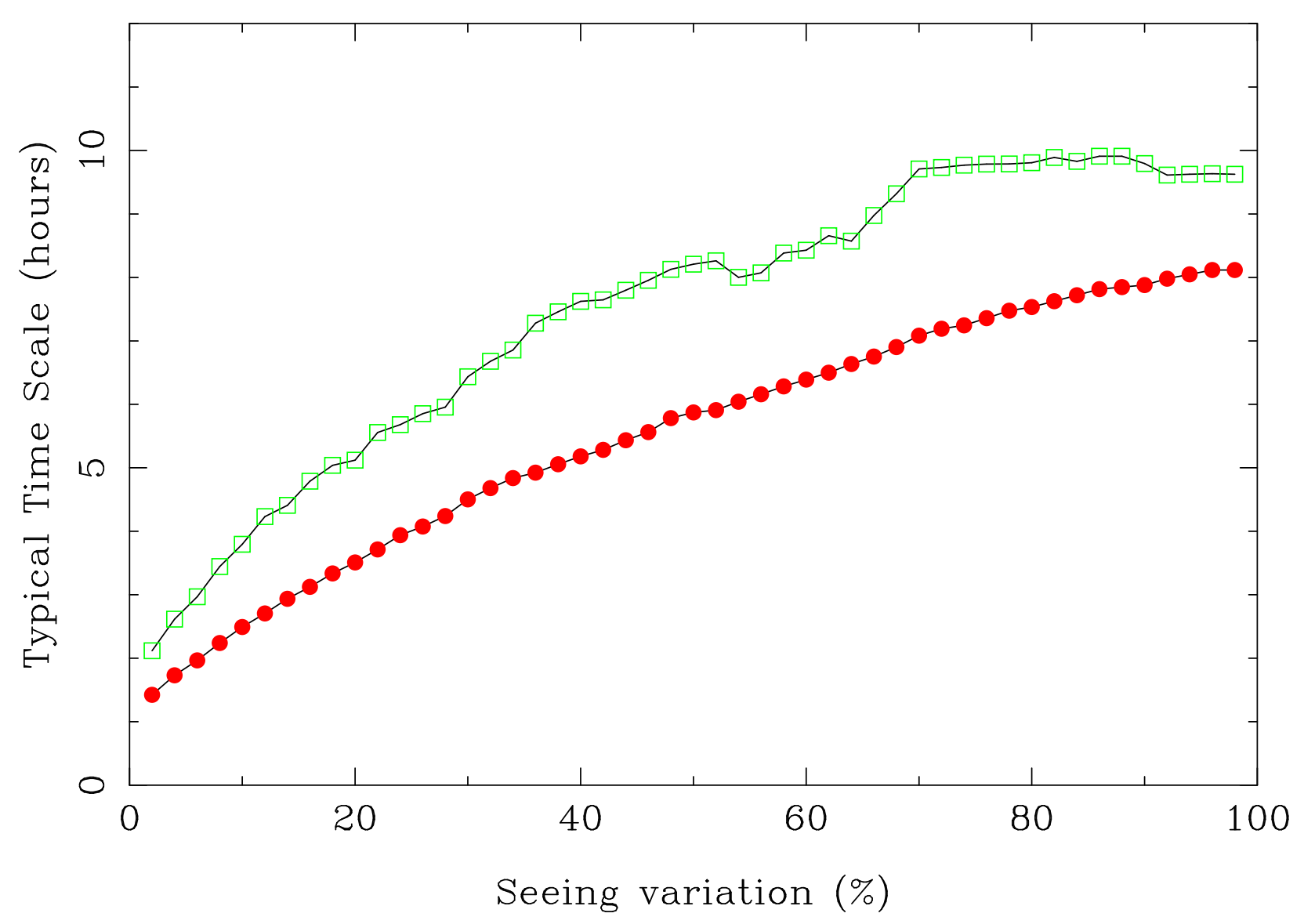

Fig. 5.- Seeing stability: Typical time scale for seeing variability within a given percentage of the reference value. The red solid circles are for any value of the reference seeing. The green symbols correspond to the cases when the reference seeing value is below $0.8^{\prime \prime}$ 


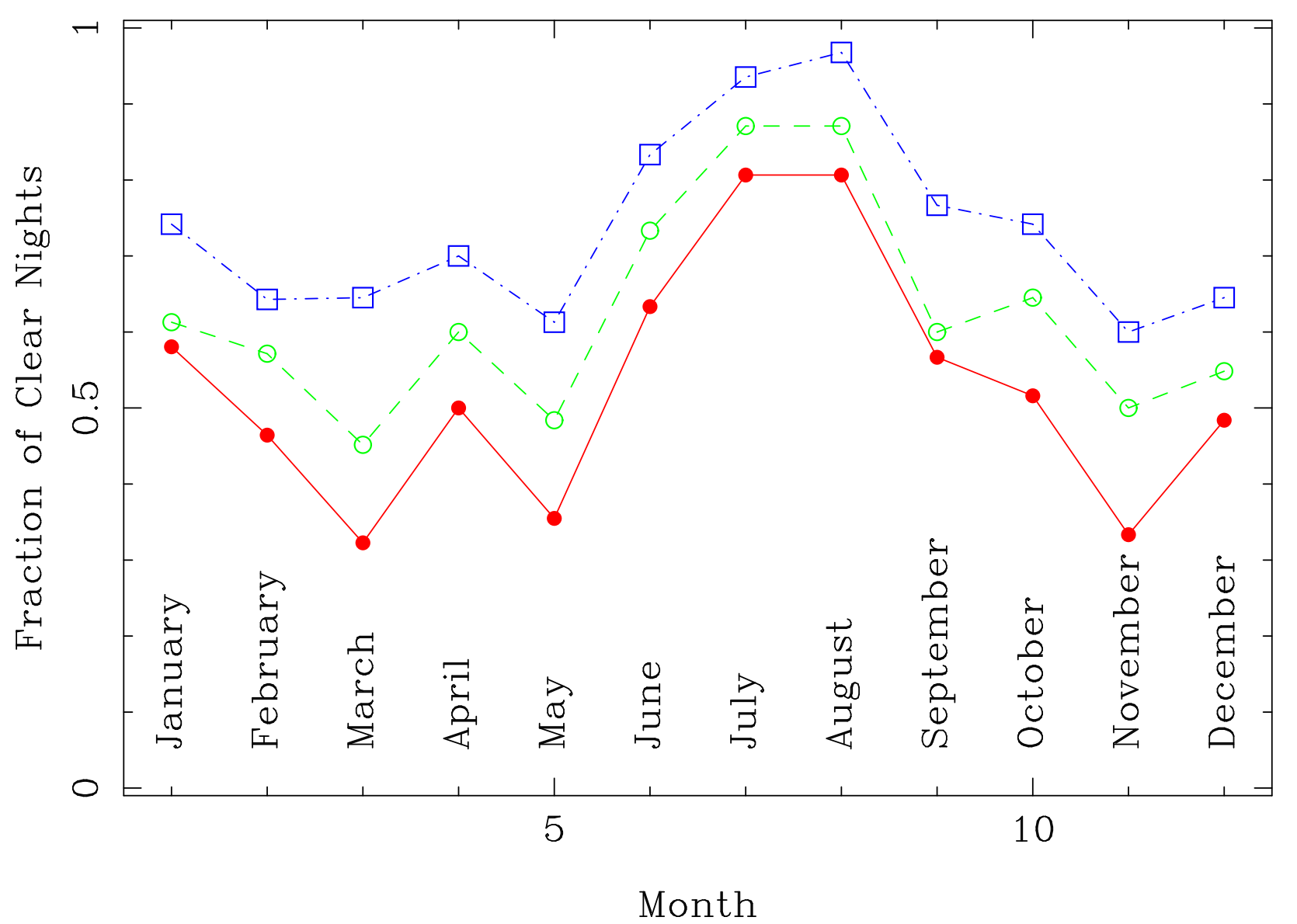

Fig. 6.- Clouds statistics along the different months for the time period 2005-2006, based on satellite images. The red-solid line and solid circles show the fraction of nights with a cloud coverage lower than $10 \%$. The green dashed-line and open circles show the fraction of nights with a cloud coverage lower than $50 \%$. Finally, the blue dashed-dotted line and open squares show the fraction of nights with a cloud coverage lower than a $70 \%$. 